\title{
A NEW TECHNIQUE FOR MAKING BRIGHT PROTON BUNCHES USING BARRIER RF SYSTEMS *
}

\author{
C. M. Bhat \\ Fermilab, Batavia, IL 60510, U.S.A. \\ (Presented at PAC2005)
}

\begin{abstract}
I describe here a very promising scheme for producing bright proton bunches for proton-antiproton and protonproton colliders. The method is based on the use of wide-band barrier rf systems. First, I explain the principle of the method. The beam dynamics simulations applied to the Fermilab Main Injector (MI) suggest that the scheme allows a wide range of bunch intensities and emittances for ppbar collider. This method has the potential to increase the instantaneous luminosity by $\geq 30 \%$ at the Tevatron.
\end{abstract}

\section{INTRODUCTION}

Producing high intensity low emittance particle bunches at hadron colliders has been one of the major problems for many years. A technique for coalescing several low intensity bunches into a high intensity bunch (using two rf systems with frequency ratio equal to an odd integer) was developed at Fermilab [1] and has been used over the last two decades. A number of other accelerator specific techniques and their operational capabilities have also been studied [2,3] elsewhere. Recently, the use of broad band rf systems [4] at synchrotrons and their potential to produce high intensity bunches for collider operation have been realized [5]. In this paper, I present a new technique [6] which uses a wide-band rf system to produce low emittance bright proton bunches. The method presented here is first of its kind.

\section{THE PRINCIPLE}

For particle beams in a synchrotron, the phase space density of the particles is highest in the vicinity of the synchronous energy. This led to the idea that an efficient way to produce a bright beam bunch is by selectively isolating the dense region of many bunches and merge them together. Recently, I have developed a technique for $(\Delta E, \Delta t)$-phase space manipulation, called, "longitudinal momentum mining" [7] for isolating dense region of particle beam in a storage ring. This method has been adopted in the Recycler [8] at Fermilab with remarkable success. The new bunch coalescing technique described here is a spin-off of the longitudinal momentum mining.

The principle of the technique is schematically illustrated in Fig. 1 for four low intensity bunch barrier coalescing. I assume that the coalescing is carried out

\footnotetext{
* Operated by Universities Research Association, Inc. for the U.S. Department of Energy under contract DE-AC02-76CH03000.

"cbhat@fnal.gov
}

above the transition energy of the synchrotron. The first step of the rf manipulation is to transfer the bunches to four rectangular "Mini Barrier" buckets of same period as that of the low intensity bunches so that the bunch centres match with the bucket centres. These mini-barrier buckets are populated in an anti-bucket (made up of two "Large Barriers" as shown in Fig. 1(a)). This transfer need not be a "matched transfer." The high momentum particles not fitting into the barrier bucket will be drifting to the leftside or to right-side, as shown in Fig. 1(a), depending on their energy relative to the synchronous energy. These particles are removed quickly from the region of interest as they hit the anti-bucket.

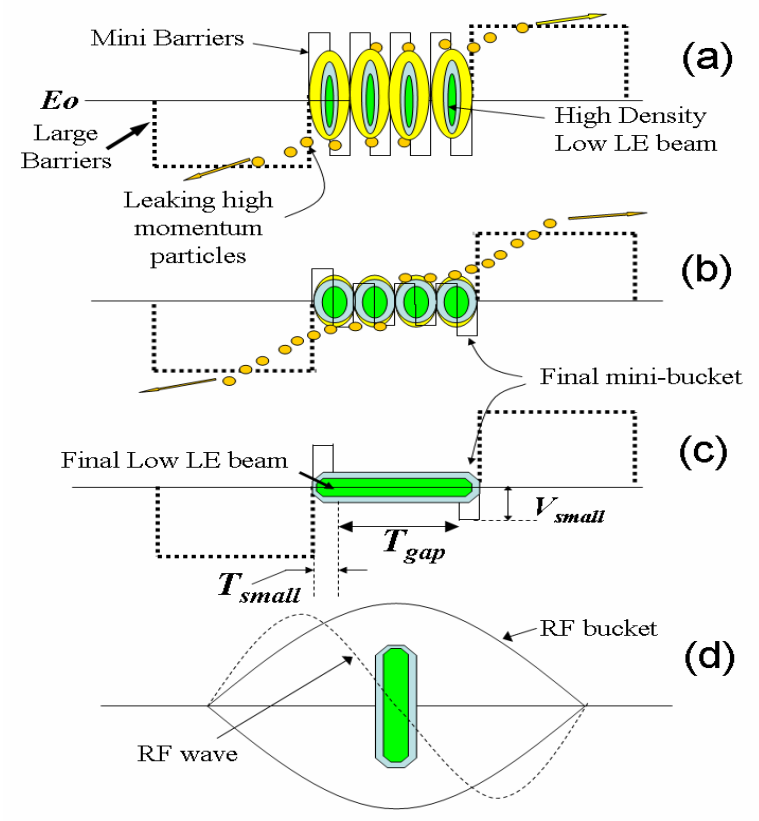

Figure 1: A schematic of barrier coalescing scheme to produce a bright bunch: a) four bunches in rectangular barrier buckets embedded between two large barriers (anti-bucket), b) intermediate step: pulse heights of minibuckets are reduced and more particles in high momentum region relative to the synchronous particles are removed, c) low LE beam bunch in barrier bucket and d) bunch after rotation in a lower harmonic sinusoidal if bucket; the rf wave is shown by dashed line.

The second step is to establish final bunch area of the bright beam by reducing adiabatically the pulse heights of 
the extreme mini-pulses to $V_{\text {small }}$ and the rest of the intermediate barrier pulse heights to zero amplitude as shown in Figs. 1(b) and 1(c). During this process more high momentum particles are removed. The longitudinal phase-space area (LE) of the final bunch is given by [9],

$$
\varepsilon_{\text {small }}=\frac{4 T_{0}|\eta| \Delta E_{\text {small }}^{3}}{\left(3 \beta^{2} E_{0} e V_{\text {Small }}\right)}+2 T_{\text {gap }} \Delta E_{\text {small }}
$$

where half-energy spread,

$$
\Delta E_{\text {small }}=\sqrt{\frac{2 \beta^{2} e V_{\text {small }} T_{\text {small }} E_{0}}{T_{0}|\eta|}}
$$

The quantities $\eta, T_{0}$ and $\beta$ are the phase slip factor, the revolution period and the ratio of the particle velocity to that of light, respectively. $E_{0}$ and $T_{\text {small }}$ are the synchronous energy of the beam particles and the barrier pulse width of the small barrier bucket, respectively (as shown in Fig. 1c). $e$ is the electron charge.

In many applications one may have to accelerate the bunch further. An example of transferring the final bunch to a sinusoidal bucket prior to further acceleration is shown in Fig. 1(d).

One can think of numerous variations of the technique described above. I have explained another method in ref. 6. One can choose one of the alternative methods depending on their merits for a specific application.

\section{MULTI-PARTICLE BEAM DYNAMICS SIMULATIONS}

To demonstrate the feasibility of the technique I have carried out beam dynamics simulations for the Main Injector (MI) machine parameters [10] using the simulation code ESME [11] for proton beam. The MI is an 8-150 GeV proton and antiproton synchrotron and is used as a $150 \mathrm{GeV}$ injector to the Tevatron. MI has many resonant and barrier rf systems. Nine to fifteen $53 \mathrm{MHz}$ bunches of proton beam are injected from the Fermilab Booster to $\mathrm{MI}$ at $8 \mathrm{GeV}$ and are accelerated to $27 \mathrm{GeV}$ (slightly above the MI transition energy of $20.49 \mathrm{GeV}$ ). The LE of the proton bunches at injection are assumed to be in the range of $0.1-0.3 \mathrm{eVs}$. The barrier $\mathrm{rf}$ manipulations are carried out at a constant energy of 27 $\mathrm{GeV}$ and the final bright bunch is transferred to $53 \mathrm{MHz}$ bucket and accelerated further before transfer to the Tevatron for proton-antiproton collider store. The proton bunch intensity specification in the Tevatron at $1 \mathrm{TeV}$ is $27 \times 10^{10} \mathrm{ppb}$ with $<2.5 \mathrm{eVs}$. In the present mode of collider operation [12], a total of 36 proton bunches are injected to the Tevatron.
The results of simulations for a train of nine bunches, each with about $0.15 \mathrm{eVs}$ and $6 \times 10^{10} \mathrm{ppb}$ are shown in Fig. 2. The distribution of the particles in a bunch at $8 \mathrm{GeV}$ is assumed to be either Gaussian or parabolic. At $27 \mathrm{GeV}$ the $53 \mathrm{MHz}$ rf voltage is reduced adiabatically from $0.5 \mathrm{MV}$ to $10 \mathrm{kV}$. Fig. 2(a) shows the final distribution. The half energy height $\Delta E$ of such bunches is about $11.5 \mathrm{MeV}$. These bunches are transferred to nine mini-barrier buckets quickly. The pulse height, pulse width and bucket area of each of the mini-buckets is chosen to be about $1.5 \mathrm{kV}, 9.5$ $\mathrm{nsec}$ and $0.22 \mathrm{eVs}$, respectively. The corresponding halfenergy spread of the mini-barrier bucket is $8.6 \mathrm{MeV}$.

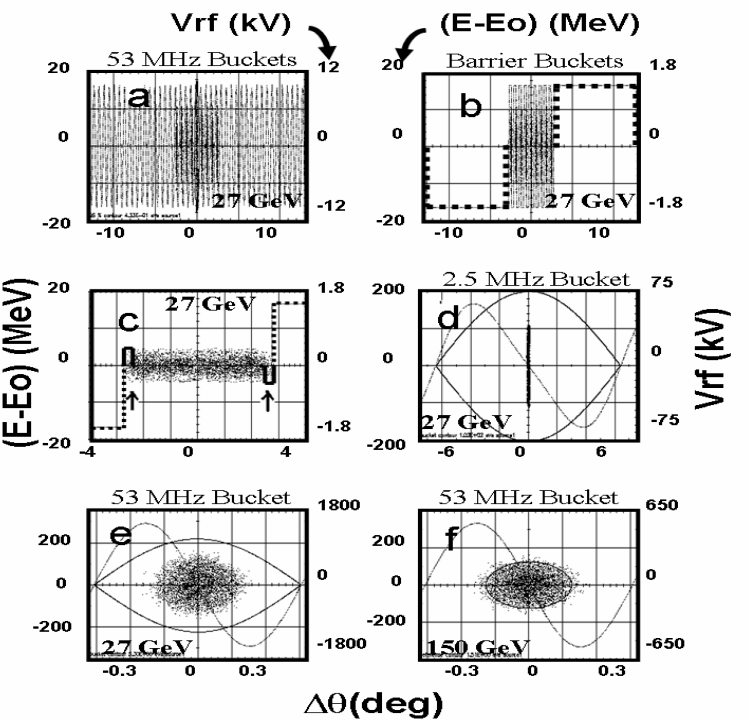

Figure 2: Simulated phase-space distribution for barrier bucket coalescing scheme using ESME. For details see the text.

The particles with momentum spread between $8.6 \mathrm{MeV}$ to 11.5 MeV escape from the mini-bucket contours and start drifting towards the large anti-barriers indicated in Figs. $2 \mathrm{~b}$ and $2 \mathrm{c}$. The time required for a particle (with energy deviation $\Delta E$ ) to drift a distance of $T_{\text {gap }}$ is

$$
T_{\text {Drift }}=\frac{T_{\text {gap }} \beta^{2} E_{0}}{|\eta| \Delta E}
$$

The particles with energy deviation $\geq 8.6 \mathrm{MeV}$ are removed in about $0.57 \mathrm{sec}$.

In the next 3 seconds the barrier rf voltage for the intermediate mini-barrier buckets is turned off slowly and linearly while reducing the last small barriers on both sides to about $400 \mathrm{~V}$. The area of the barrier bucket embedded between large barriers, shown in Fig. 2(c), is about 1.5 eVs. The simulation showed that about $87 \%$ of beam particles can be captured in the small barrier bucket. The bunch length is about $200 \mathrm{~ns}$ and half energy height $\Delta E$ is about $4.5 \mathrm{MeV}$. Such a bunch is ideal for $53 \mathrm{MHz}$ capture after a quarter synchrotron rotation in $2.5 \mathrm{MHz}$ rf bucket 
as shown in Fig. 2(d). The captured bunch in $53 \mathrm{MHz}$ bucket at $27 \mathrm{GeV}$ and the one accelerated to $150 \mathrm{GeV}$ are shown in Figs. 2(e) and 2(f), respectively. LE (95\%) at $150 \mathrm{GeV}$ is found to be $1.6 \mathrm{eVs}$. If we begin with about $5 \times 10^{10} \mathrm{ppb}$ at $8 \mathrm{GeV}$ (LE 0.15 eVs/53 MHz bunch) the final expected bunch intensity is about $39 \times 10^{10} \mathrm{ppb}$ which is about $44 \%$ larger than the design bunch intensity for the collider operation. The total time required for the $27 \mathrm{GeV}$ barrier coalescing is about $4.5 \mathrm{sec}$.

\section{What happens to the escaped High Momentum beam particles?}

For the scheme described above, about $13 \%$ of the beam particles are found to escape during barrier coalescing at $27 \mathrm{GeV}$. They will come back only after about $7 \mathrm{sec}$. During the coalescing, these particles are found to be still drifting away from the mini-bucket. After the $53 \mathrm{MHz}$ capture at $27 \mathrm{GeV}$, majority of these particles are captured and get accelerated along with the bunch of interest. The rest of the beam particles, not captured in the $53 \mathrm{MHz}$ bucket, are lost during acceleration to $150 \mathrm{GeV}$. In any case, the region in the vicinity of the high intensity bunch is cleared off of any beam particles.

\section{Wake-field and space-charge effects}

The MI has many rf systems, several injection and extraction regions. Each of these contributes to the beam machine impedance. A first attempt to address these effects including space-charge effect on producing high intensity low emittance proton bunches is done in ref. 6 . We conclude that none of the above issues are of concern to adopt the barrier bucket coalescing scheme in the MI.

The experimental investigation of the technique for proton coalescing in the MI is in progress.

\section{LUMINOSITY POTENTIAL OF THE SCHEME FOR THE TEVATRON}

The instantaneous luminosity of the Tevatron depends on the longitudinal emittance of the bunches through hourglass factor (it is a weak dependence, but, increases with smaller LE), varies inversely with the transverse emittance. Typically the transverse profile of the proton beam in the MI is Gaussian in shape. A beam bunch with $39 \times 10^{10} \mathrm{ppb}$ and a transverse emittance of $16 \pi-\mathrm{mm}-\mathrm{mr}$ can be tapered transversely to achieve the required intensity of $27 \times 10^{10} \mathrm{ppb}$. This gives a reduction in transverse emittance by a factor of $\sim 4$. Beam-beam interaction and other beam phenomenon demand that the proton transverse emittance be similar to that of pbars $(\sim 10$ $\pi$-mm-mr) in the Tevatron. Taking into account these effects one might expect up to about $>50 \%$ increase in the peak luminosity without any further increase in the pbar intensities.

\section{SUMMARY}

I explain a new scheme to produce low emittance high intensity bunches for hadron colliders which uses wide band barrier rf system. This is a very promising scheme and, can be adopted at proton- proton collider and proton-antiproton collider to improve the beam quality and hence the luminosity. I have explained the general principle of the technique and detailed simulation results for one of many possibilities. It has a potential of increasing the peak-luminosity by at least $50 \%$ at the Fermilab Tevatron.

I would like to thank Jim MacLachlan, Bill Foster, Dave Wildman, Pushpa Bhat, Dave Johnson, Valeri Lebedev and Vladimir Shiltsev for many useful discussions.

\section{REFERENCES}

[1] J. E. Griffin, J. A. MacLachlan and Z. B. Qian, IEEE, Trans. Nucl., Sci., NS-30, No. 4, (1983) 2627.

[2] J. M. Brennan et al, Proc. of EPAC2004, Lucerne, Switzerland (2004) p 905.

[3] H. Damerau and R. Groby, Proc. of EPAC2004, Lucerne, Switzerland (2004) p 1852.

[4] J. E. Griffin et al, IEEE, Trans. Nucl., Sci., NS-30 (1983) 3502

[5] Initial ideas for use of broad band rf system to produce bright proton bunches were proposed by the author and by Bill Foster independently (after the installation of broad-band rf systems in the MI).

[6] C. M. Bhat, Fermilab-FN-0761 (2004).

[7] C. M. Bhat, Phys. Letts. A330 (2004) 481.

[8] G. Jackson, PAC2003, (2003) p 3159.

[9] S.Y. Lee and K. Y. Ng, Phys. Rev. E, Vol. 55 (1997) 5992.

[10] "Fermilab Main Injector Technical Design Handbook” (Internal Report, 1994).

[11] J. A. MacLachlan, HEACC'98, Int. Conf. on High Energy Accelerators, Dubna, Sept. 1998; J. A. MacLachlan, Fermilab Report No FN-529, (1989) (private communications 2003), http://wwwap.fnal.gov/ESME/.

[12] P. C. Bhat and W. J. Spalding, Hadron Collider Physics, HCP2004, edited by H. Weerts, AIP Proceedings Vol.753 (2004) p 30. 